\title{
On minimal time of process of circuit optimisation
}

\author{
A.M. Zemliak \\ Autonomous University of Puebla, Mexico, Puebla \\ National Technical University of Ukraine, Ukraine, Kiev. azemliak@mail.ru
}

\begin{abstract}
The process of analogue circuit optimisation is mathematically defined as a controllable dynamic system. In this context the minimisation of the processor time of designing can be formulated as a problem of time minimisation for transitional process of dynamic system. A special control vector that changes the internal structure of the main equations of optimisation procedure serves as a principal tool for searching the best strategies of the optimisation process. The creation of the best strategy of the optimisation having the minimum processor time leads to search of the structure of control vector providing the minimum of a special functional that can be defined as CPU time. In this case a well-known maximum principle of Pontryagin is the best theoretical approach for finding of the optimum structure of control vector. Practical approach for realization of the maximum principle is based on the analysis of behavior of a Hamiltonian for various strategies of optimisation. The possibility of applying the maximum principle to the problem of optimisation of electronic circuits is analyzed in detail. It is shown that in spite of the fact that the problem of optimisation is formulated as a nonlinear task, and the maximum principle in this case isn't a sufficient condition for obtaining a minimum of the functional, it is possible to obtain the decision in the form of local minima. The relative acceleration of the CPU time for the best strategy found by means of maximum principle compared with the traditional approach is equal two to three orders of magnitude. A new theoretical result is obtained.
\end{abstract}

Keywords - circuit optimisation, controllable dynamic system, optimisation strategies, maximum principle of Pontryagin.

\section{INTRODUCTION}

To improve the overall quality of electronic circuit designs, it is very important to reduce their design time. Many works devoted to this problem focus on how to reduce the number of operations when solving two main problems: circuit analysis and numerical optimisation. By solving these problems successfully, one can reduce the total time required for analog circuit optimisation and this fact serves as a basis for improving design quality.

The methods used to analyse complex systems are being improved continuously. Some well-known ideas related to the use of a method of sparse matrixes [1], [2] and decomposition methods [3]-[5] are used for the reduction of time for the analysis of circuits. Some alternative methods such as homotopy methods [6] were successfully applied to circuit analysis.
Practical methods of optimisation were developed for circuit designing, timing, and area optimisation [7], [8]. However, classical deterministic optimisation algorithms may have a number of drawbacks: they may require that a good initial point be selected in the parameter space, they may reach an unsatisfactory local minimum, and they require that the cost function be continuous and differentiable. To overcome these issues, special methods were applied to determine the initial point of the process by centering [9] or applying geometric programming methods [10].

A more general formulation of the circuit optimisation problem was developed on a heuristic level some decades ago [11]. This approach ignored Kirchhoff's laws for all or part of a circuit during the optimisation process. The practical aspects of this idea were developed for the optimisation of microwave circuits [12] and for the synthesis of high-performance analog circuits [13] in an extreme case where all the equations of the circuit were not solved during the optimisation process.

In work [14] the problem of circuit optimisation is formulated in terms of the theory of optimal control. Thus, the process of circuit optimisation was generalized and defined as the dynamic controllable system. In this case, the basic element is the control vector that changes the structure of the equations of the system of optimisation process. Thus, there is a set of strategies of optimisation that have different number of operations and different computing times. The introduction and analysis of the function of Lyapunov of the optimisation process [15], [16] allows comparison of various strategies of optimisation and choosing the best of them having minimum processor time. At the same time, the problem of searching for the optimal strategy and the corresponding optimal trajectory can be solved most appropriately within the maximum principle of Pontryagin [17].

The main complexity of application of the maximum principle consists of the search of initial values for auxiliary variables at the solution of the conjugate system of equations. Application of the maximum principle in case of linear dynamic systems is based on the creation of an iterative process [18], [19].

In case of nonlinear systems, the convergence of this process is not guaranteed. However, application of the additional approximating procedures [20], [21] allows constructing sequence of the solutions converging to a limit under certain conditions. 
The first step in the problem of possibility of application of maximum principle for circuit optimisation was presented in [22]. In the present work, the solution of the problem is presented for $\mathrm{N}$-dimensional case.

\section{THEORETICAL BASIS}

The following question is interesting, whether it is possible to extend the obtained analytical result to the numerical solution of the optimisation problem for nonlinear circuit of any dimension. Let's consider the problem of optimisation of a nonlinear circuit with two nodes shown in Fig. 1.

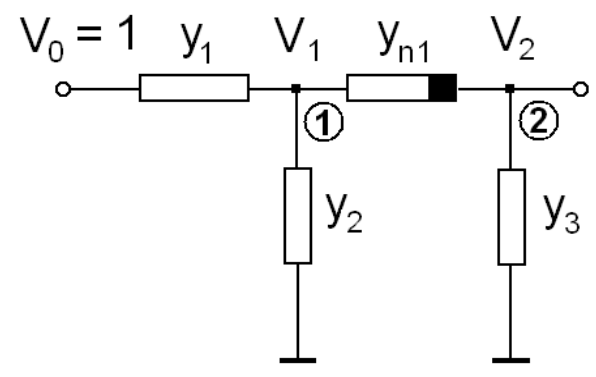

Fig. 1. Nonlinear two-node voltage divider

The given circuit is a nonlinear divider of voltage. There are three independent parameters $(K=3)$ and two dependent ones $(M=2)$. The nonlinear element has the following dependency: $y_{n 1}=y_{0}+a\left(V_{1}-V_{2}\right)^{2}$. We define the voltage $V_{0}$ as 1 , and the variables $x_{1}, x_{2}, x_{3}, x_{4}$, and $x_{5}$ as: $x_{1}^{2}=y_{1}, x_{2}^{2}=y_{2}, x_{3}^{2}=y_{3}, x_{4}=V_{1}$, and $x_{5}=V_{2}$. The vector of the phase variables of the circuit is $X \in R^{5}$. In this case the nonlinear element is defined by the following expression: $y_{n 1}=y_{0}+a\left(x_{4}-x_{5}\right)^{2}$.

Let's determine cost function of process of optimization by the formula:

$$
C(\mathbf{X})=\left(x_{5}-w\right)^{2}
$$

where $w$ - the required value of output voltage. Following theoretical bases that were developed in [14], we formulate the problem for circuit optimisation as a task of search of the optimisation strategy with minimum possible CPU time. For this purpose, we define the functional, which is subject to minimisation, by the following expression:

$$
J=\int_{0}^{T} f_{\mathrm{o}}(\mathbf{X}) d t
$$

where $f_{0}(\mathbf{X})$ is the function that is conditionally determining the density of number of arithmetic operations in a unit of time $t$. In that case, the integral (2) defines total number of operations necessary for circuit optimisation and is proportional to the total CPU time.

The structure of function $f_{0}(\mathbf{X})$ cannot be defined. However, we can compute CPU time using the capabilities of the compiler. We will further identify the integral (2) with CPU time, and therefore, the problem of minimisation of CPU time corresponds to a problem of minimisation of the integral (2).

The model of a circuit is defined by the following system:

$$
\begin{aligned}
& g_{1}(\mathbf{X}) \equiv\left(1-x_{4}\right) x_{1}^{2}-\left\lfloor y_{0}+a\left(x_{4}-x_{5}\right)^{2}\right\rfloor\left(x_{4}-x_{5}\right)-x_{4} x_{2}^{2}=0 \\
& g_{2}(\mathbf{X}) \equiv\left\lfloor y_{0}+a\left(x_{4}-x_{5}\right)^{2}\right\rfloor\left(x_{4}-x_{5}\right)-x_{5} x_{3}^{2}=0
\end{aligned}
$$

The system of equations of the optimisation procedure can be presented in the following form:

$$
\frac{d x_{i}}{d t}=f_{i}(\mathbf{X}, \mathbf{U}), \quad i=1,2, \ldots, N
$$

where

$$
f_{i}(\mathbf{X}, \mathbf{U})=-\frac{\delta}{\delta x_{i}} F(\mathbf{X}, \mathbf{U}), i=1,2, K
$$

$$
f_{i}(\mathbf{X}, \mathbf{U})=-u_{i-K} \frac{\delta}{\delta x_{i}} F(\mathbf{X}, \mathbf{U})+\left(1-u_{i-K}\right) \alpha_{i}, i=K+1, \ldots, N
$$

where $F(\mathbf{X}, \mathbf{U})$ is the generalized cost function of the optimisation process defined by expression:

$$
F(\mathbf{X}, \mathbf{U})=C(\mathbf{X})+\sum_{j=1}^{M} u_{j} g_{j}^{2}(\mathbf{X}),
$$

$\alpha_{i}$ for $i=K+1, \ldots, N$ is the additional parameter defining an increment of the value of the dependent variables in the course of optimization and computed by the formula:

$$
\alpha_{i}=\frac{1}{\tau}\left[\eta_{i}\left(\mathbf{X}^{s+1}\right)-x_{i}^{s}\right],
$$

and $\eta_{i}(\mathbf{X})$ is the implicit function defining the component number $i$ of a vector $\mathbf{X}$ at the solution of system (3), $\tau$ is a step of integration and $s$ is the step number of the procedure of the integration of system (4).

Let's obtain the main expressions corresponding to the maximum principle. The conjugate system of the equations for the additional variables $\psi_{i}$ has a form:

$$
\frac{d \psi_{i}}{d t}=-\sum_{k=1}^{N} \frac{\partial f_{k}(\mathbf{X}, \mathbf{U})}{\partial x_{i}} \cdot \psi_{k}
$$

The Hamiltonian is determined by the following formula:

$$
\begin{aligned}
H(\mathbf{X}, \boldsymbol{\Psi}, \mathbf{U}) & =\sum_{i=1}^{N} \psi_{i} \cdot f_{i}(\mathbf{X}, \mathbf{U}) \\
& =\sum_{i=1}^{K} \psi_{i} \cdot f_{i}(\mathbf{X}, \mathbf{U})+\sum_{i=K+1}^{N} \psi_{i} \cdot f_{i}(\mathbf{X}, \mathbf{U})
\end{aligned}
$$


where the first and second sums are defined by the following expressions:

$$
\begin{aligned}
\sum_{i=1}^{K} \psi_{i} \cdot f_{i}(\mathbf{X}, \mathbf{U})=-\sum_{i=1}^{K} \psi_{i} \cdot \frac{\delta C}{\delta x_{i}}-\sum_{i=1}^{K} \psi_{i} \sum_{k=1}^{M} u_{k} \cdot \frac{\delta\left(g_{k}(\mathbf{X})\right)^{2}}{\delta x_{i}} \\
\sum_{i=K+1}^{N} \psi_{i} \cdot f_{i}(\mathbf{X}, \mathbf{U})=\sum_{i=K+1}^{N}\left(1-u_{i-K}\right) \psi_{i} \alpha_{i} \\
-\sum_{i=K+1}^{N} u_{i-K} \psi_{i}\left[\frac{\delta C}{\delta x_{i}}+\sum_{k=1}^{M} u_{k} \frac{\delta\left(g_{k}(\mathbf{X})\right)^{2}}{\delta x_{i}}\right]
\end{aligned}
$$

As a result the Hamiltonian can be expressed as follows:

$$
H(\mathbf{X}, \boldsymbol{\Psi}, \mathbf{U})=h_{c}+h_{0}+h_{1}+h_{2}
$$

where $h_{\mathrm{c}}$ is the part of a Hamiltonian that does not depend on the control vector,

$$
h_{c}(\mathbf{X}, \mathbf{\Psi})=-\sum_{i=1}^{K} \psi_{i} \cdot \frac{\delta C}{\delta x_{i}}+\sum_{i=K+1}^{N} \psi_{i} \alpha_{i}
$$

Other components of Hamiltonian depend on the control vector $\mathbf{U}$ :

$$
\begin{gathered}
h_{0}(\mathbf{X}, \boldsymbol{\Psi}, \mathbf{U})=-\sum_{i=K+1}^{N} u_{i-K} \psi_{i} \alpha_{i}, \\
h_{1}(\mathbf{X}, \boldsymbol{\Psi}, \mathbf{U})=-\sum_{i=1}^{K} \psi_{i} \sum_{k=1}^{M} u_{k} \cdot \frac{\delta\left(g_{k}(\mathbf{X})\right)^{2}}{\delta x_{i}}, \\
h_{2}(\mathbf{X}, \boldsymbol{\Psi}, \mathbf{U})=-\sum_{i=K+1}^{N} u_{i-K} \psi_{i}\left[\frac{\delta C}{\delta x_{i}}+\sum_{k=1}^{M} u_{k} \frac{\delta\left(g_{k}(\mathbf{X})\right)^{2}}{\delta x_{i}}\right] .
\end{gathered}
$$

Let's designate the sum of these three components as $h_{v}(\mathbf{X}, \boldsymbol{\Psi}, \mathbf{U})\left(h_{\mathrm{v}}=h_{0}+h_{1}+h_{2}\right)$. Formulas (1)-(15) define the process of system optimisation and the process of computing a Hamiltonian in case of $K$ independent variables and $M$ dependent variables $(N=K+M)$. In the case of the circuit presented in Fig. 1, formulas are used for $K=3$ and $M=2$. In this case the control vector of $\mathbf{U}$ contains two components $\left(u_{1}, u_{2}\right)$. The supremum of the function $h_{v}(\mathbf{X}, \boldsymbol{\Psi}, \mathbf{U})$ in the parameter $\mathbf{U}$ will be designated $H_{\max }$ :

$$
H_{\max }(\mathbf{X}, \boldsymbol{\Psi})=\sup _{u \in \mathbf{U}} h_{v}(\mathbf{X}, \boldsymbol{\Psi}, \mathbf{U})
$$

For the circuit in Fig. 1 this function is defined by the following expression:

$$
H_{\max }(\mathbf{X}, \boldsymbol{\Psi})=\max \left\{\begin{array}{l}
h_{v}(\mathbf{X}, \boldsymbol{\Psi},(0,0)), h_{v}(\mathbf{X}, \boldsymbol{\Psi},(0,1)), \\
h_{v}(\mathbf{X}, \boldsymbol{\Psi},(1,0)), h_{v}(\mathbf{X}, \boldsymbol{\Psi},(1,1))
\end{array}\right\} .
$$

The structure of the control vector providing this maximum in each point of optimisation process represents the result of the use of the maximum principle.

\section{NUMERICAL RESULTS}

The analysis of the process of optimisation for a circuit with two nodes allows for the finding of the optimum structure of the control vector. The possibility of applying the maximum principle of Pontryagin to the problem of optimisation of electronic circuits is analysed. It is shown that in spite of the fact that the problem of optimisation is formulated as a nonlinear task, and the maximum principle in this case isn't a sufficient condition for obtaining a maximum of the functional, it is possible to obtain the decision in the form of local minima. Local minima of the functional, which is defined as the processor time necessary for the procedure of optimisation, provide a rather low value of the functional. The relative acceleration of the CPU time for the best strategy found by means of maximum principle compared with the traditional approach is equal two to three orders of magnitude.

The behaviour of Hamiltonian for four possible options of the control vector $\mathbf{U}$ : (00), (01), (10), and (11) with the correct initial value of an auxiliary vector $\boldsymbol{\Psi}$, $\left(\boldsymbol{\Psi}_{0 c}=(0.3,-1.85,-0.35,-1.9,0.32,2.5)\right)$ is presented in Fig. 2.

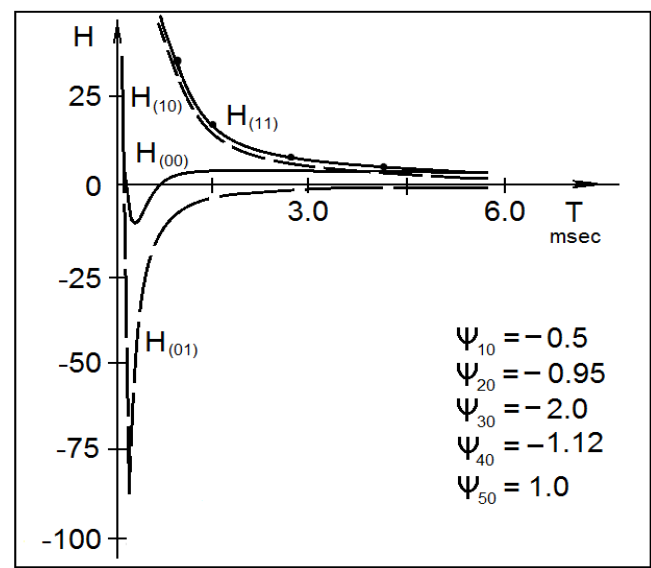

Fig. 2. Time dependency of functions $H_{(00)}, H_{(01)}, H_{(10)}, H_{(11)}$ for correct value of parameter $\boldsymbol{\Psi}_{0 c}$

The value of $\boldsymbol{\Psi}_{0 c}$ has been obtained by the additional optimising procedure on the basis of a gradient method for the following initial point in process of designing $\mathbf{X}^{0}$ : $\left(x_{10}=1.0, x_{20}=1.0, x_{30}=1.0, x_{40}=-1.5, x_{50}=-1.6\right.$ ). Four possible combinations of the components of the control vector $\mathbf{U}$ define four various dependencies for Hamiltonian: $\left(\boldsymbol{H}_{(\mathrm{OO})}, \boldsymbol{H}_{(\mathrm{O})}, \boldsymbol{H}_{(10)}, \boldsymbol{H}_{(11)}\right)$. The Hamiltonian corresponding to the control vector (11) has the greatest value of all possible. Therefore, the optimum trajectory corresponds to this vector and defines the first part of a trajectory in the space of parameters. Some twodimensional projections of a trajectory of optimisation process in the space of variables $\mathbf{X}$ are presented in Fig. 3. 


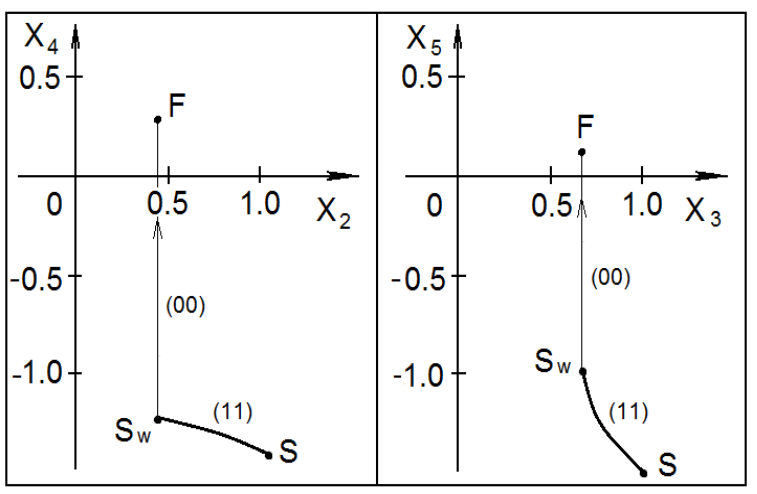

Fig. 3. Projections of trajectory of optimisation process with initial point $\mathbf{X}^{\mathbf{0}}:\left(x_{10}=1, x_{20}=1, x_{30}=1, x_{40}=-1.5, x_{50}=-1.6\right)$

A part of the trajectory from point $S$ to point $S_{\mathrm{w}}$ corresponds to the control vector $\mathbf{U}=(11)$. Then, at a time point of $5.753 \mathrm{msec}$, which corresponds to 121 steps of integration of the system (4), the Hamiltonian corresponding to the strategy with the control vector (00) becomes the greatest of all, and at this moment the vector (00) becomes the optimum control vector. The trajectory, including the jump and the current point of the optimisation process, instantly moves to the final point of the solution of a problem of $F$.

The data of optimisation process for the presented circuit for four strategies of structural basis and the same initial point $\mathbf{X}^{0}$ are provided in Table 1 for comparison.

Table 1

Data of all strategies of structural basis

\begin{tabular}{|r|r|r|r|}
\hline $\mathrm{N}$ & $\begin{array}{l}\text { Control } \\
\text { vector }\end{array}$ & $\begin{array}{l}\text { Iterations } \\
\text { number }\end{array}$ & $\begin{array}{l}\text { Total CPU } \\
\text { time }(\mathrm{sec})\end{array}$ \\
\hline 1 & $\left(\begin{array}{ll}0 & 0\end{array}\right)$ & 116973 & 16.081 \\
\hline 2 & $\left(\begin{array}{ll}0 & 1\end{array}\right)$ & 139143 & 8.897 \\
\hline 3 & $\left(\begin{array}{ll}1 & 0\end{array}\right)$ & 133154 & 11.241 \\
\hline 4 & $\left(\begin{array}{ll}1 & 1\end{array}\right)$ & 170953 & 7.934 \\
\hline
\end{tabular}

The most rapid strategy is MTSO, which corresponds to the control vector (11), has processor time of $7.934 \mathrm{sec}$. The optimum strategy considered above and found by the maximum principle has a gain in time of 1379 times that of strategy (11) and 2795 times that of the TSO with $\mathbf{U}=(00)$.

The behaviour of the Hamiltonian that corresponds to another choice for the initial point of optimisation process of circuit $\mathbf{X}^{0}:\left(x_{10}=1.0, x_{20}=1.0, x_{30}=1.0, x_{40}=-2.5\right.$,

$\left.x_{50}=-2.5\right)$ for four possible values of the control vector $\mathbf{U}:(00),(01),(10)$, and (11) is shown in Fig. 4.

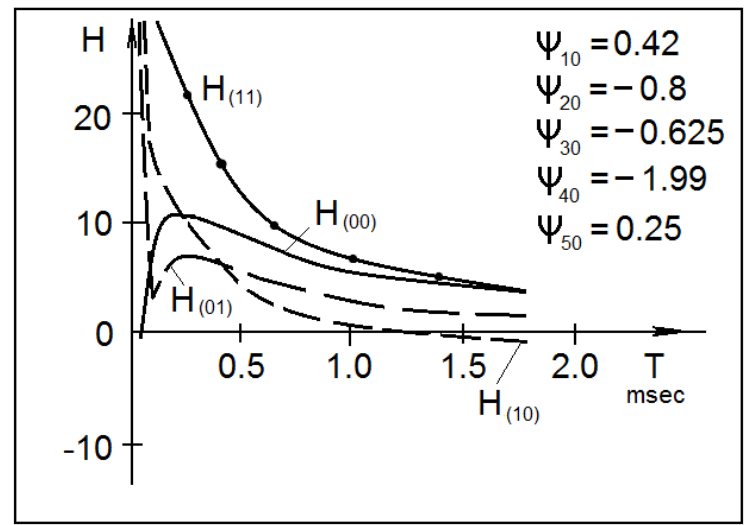

Fig. 4. Time dependency of functions $H_{(00)}, H_{(01)}, H_{(10)}, H_{(11)}$ for other correct value of parameter $\boldsymbol{\Psi}_{0 c}=(0.42,-0.8,-0.625,-1.99,0.25)$

In this case the following correct value of an auxiliary vector $\quad \Psi_{0 c}=(0.42,-0.8,-0.625,-1.99,0.25)$ providing the minimum value of processor time has been obtained. In this example, the optimisation procedure is defined by the control vector (11) from $\mathrm{T}=0$ to $\mathrm{T}=1.761 \mathrm{msec}$ because the Hamiltonian of $H_{(11)}$ has the maximum value for this control vector of the four possible. Then, at a time of $\mathrm{T}=1.761 \mathrm{msec}$, which corresponds to the 35 th step of process of integration of system (4) the Hamiltonian corresponding to the control vector $(00)$ has the maximum value $\left(H_{(00)}>=H_{(11)}\right)$ and the switching to the TSO is observed. The movement corresponding to the strategy (00) is carried out on one step of integration and the current point of the optimisation process moves to the final point of $F$ with the given accuracy. It is clear from the behaviour of the projections of the optimisation trajectory shown in Fig. 5.

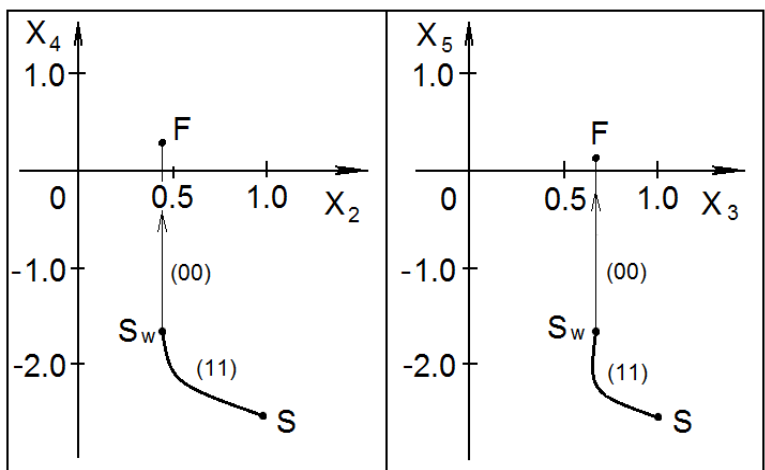

Fig. 5. Projections of trajectory of optimisation process with initial point $\mathbf{X}^{\mathbf{0}}:\left(x_{10}=1, x_{20}=1, x_{30}=1, x_{40}=-2.5, x_{50}=-2.5\right)$

It is important to emphasize that the numerical algorithm automatically switches from one strategy to another on the basis of ratio (16), corresponding to the maximum principle. It would be desirable to note that the obtained decision is not the unique local minimum of the target function of $\mathrm{T}$. Other local minimum has been reached with the other vector $\boldsymbol{\Psi}$. 
The behaviour of the function of Hamilton for the same initial point of the optimisation process of the circuit $\mathrm{X} 0$ : $\left(x_{10}=1, x_{20}=1, x_{30}=1, x_{40}=-2.5, x_{50}=-2.5\right)$

but containing other initial value for auxiliary vector $\boldsymbol{\Psi}$ is presented in Fig. 6. The correct value of $\boldsymbol{\Psi}_{0 c}$ obtained by the additional optimising procedure is next $\boldsymbol{\Psi}_{0 c}=(0.1,-0.02,-0.5,-0.2,0.6)$. In this case the other strategy is optimal one.

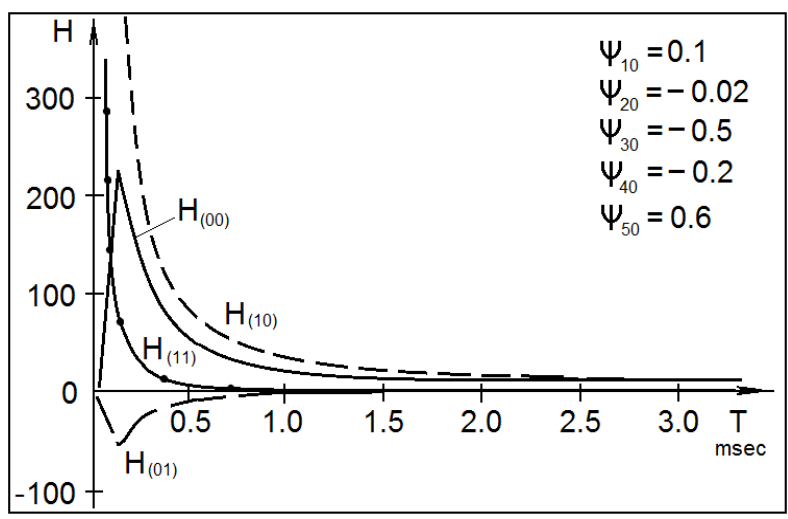

Fig. 6. Time dependency of functions $H_{(00)}, H_{(01)}, H_{(10)}, H_{(11)}$ for other correct value of parameter $\boldsymbol{\Psi}_{0 c}=(0.1,-0.02,-0.5,-0.2,0.6)$

An additional optimisation by means of parameter $\boldsymbol{\Psi}_{0}$ leads to other local minimum that is visible from the obtained dependencies. The Hamiltonian corresponding to the strategy with control vector (10) accepts the maximum value and this strategy is the first part of the optimum strategy.

The Hamiltonian corresponding to TSO with the control vector $(00)$ is greater than for all other strategies from the point corresponding to the 38th step of integration, and a switching to TSO takes place. That is also clear from the behaviour of projections of the optimisation trajectory in Fig. 7.

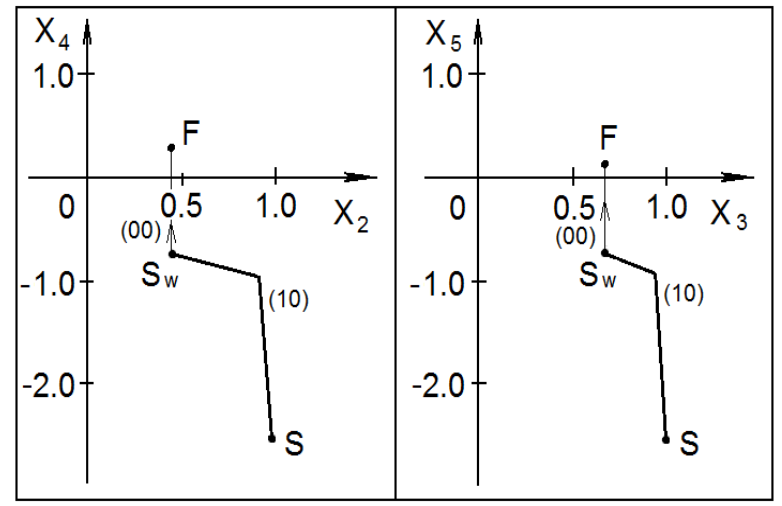

Fig. 7. Projections of trajectory of optimisation process for other correct value of $\boldsymbol{\Psi}_{0 c}=(0.1,-0.02,-0.5,-0.2,0.6)$
The total time of optimization is equal $3.345 \mathrm{msec}$. This solution represents other local minimum of the functional (2).

It is important to note that the strategy found from the conditions of the maximum principle and corresponding to the control vector with two parts (10) and (00) and switching between them on a 73rd step of integration has not been predicted in previous research. In the previous research [23] it was supposed that the optimum strategy must be constructed on the basis of the combination of MTSO and TSO. This assumption is not always carried out as shown in the present analysis.

Let's consider use of the maximum principle for the problem of optimisation of an active circuit: the onecascade transistor amplifier presented in Fig. 8.

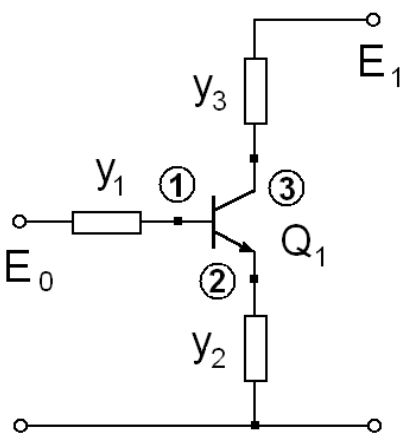

Fig. 8. One-cascade transistor amplifier

The sources of voltage of $E_{0}, E_{1}$ are defined. The model of the transistor, which is the known model of Ebers-Moll on a direct current used in the SPICE system, is chosen [24]. The purpose of optimisation of this circuit is to determine the right choice of admittances $y_{1}, y_{2}, y_{3}$ to provide the necessary values of the voltage on emitter $V_{\mathrm{EB} 0}$ and collector $V_{\mathrm{CB} 0}$ transitions of the transistor. If the current values for the voltage on transitions of the transistor are $V_{\mathrm{EB}}$ and $V_{\mathrm{CB}}$, then a natural formula for the cost function of the optimisation process in this case is as follows:

$$
C(\mathbf{X})=\left(V_{E B}-V_{E B 0}\right)^{2}+\left(V_{C B}-V_{C B 0}\right)^{2} .
$$

Minimisations of the cost function or to be exact a reduction here to zero allows for solving a problem of optimisation of a circuit. In the traditional approach to design, three conductivities $y_{1}, y_{2}, y_{3}$ are defined as independent variables $(K=3)$ and three nodal voltages $V_{1}, V_{2}, V_{3}$ are determined as dependent $(M=3)$. We will define six component of a vector of $X$ as follows:

$$
\begin{aligned}
& x_{1}^{2}=y_{1}, x_{2}^{2}=y_{2}, x_{3}^{2}=y_{3}, x_{4}=V_{1}, \\
& x_{5}=V_{2}, x_{6}=V_{3} .
\end{aligned}
$$


Cost function can be defined as:

$$
C(\mathbf{X})=\left(x_{5}-x_{4}-V_{E B 0}\right)^{2}+\left(x_{6}-x_{4}-V_{C B 0}\right)^{2} .
$$

The mathematical model of a circuit is defined by the system of three equations of Kirchhoff:

$$
\begin{aligned}
& g_{1}(\mathbf{X}) \equiv I_{B}-\left(E_{0}-x_{4}\right) x_{1}^{2}=0 \\
& g_{2}(\mathbf{X}) \equiv I_{E}-x_{2}^{2} x_{3}=0 \\
& g_{3}(\mathbf{X}) \equiv I_{C}-\left(E_{1}-x_{6}\right) x_{3}^{2}=0,
\end{aligned}
$$

where currents of base $I_{\mathrm{B}}$, emitter $I_{\mathrm{E}}$ and a collector $I_{\mathrm{C}}$ are defined in the model of Ebers-Moll.

The structural basis of optimisation strategies includes eight strategies. Results of optimisation of the presented circuit for all strategies of structural basis and a gradient method are presented in Table 2. The optimisation procedure is defined by formulas (4)-(7).

Table 2

Data of all strategies of structural basis for one-cascade transistor amplifier

\begin{tabular}{|r|r|r|r|}
\hline $\mathrm{N}$ & $\begin{array}{l}\text { Control } \\
\text { vector }\end{array}$ & $\begin{array}{l}\text { Iterations } \\
\text { number }\end{array}$ & $\begin{array}{l}\text { Total CPU } \\
\text { time }(\mathrm{sec})\end{array}$ \\
\hline 1 & $\left(\begin{array}{llll}0 & 0 & 0\end{array}\right)$ & 6363 & 2,81 \\
\hline 2 & $\left(\begin{array}{lll}0 & 0 & 1\end{array}\right)$ & 36556 & 12,63 \\
\hline 3 & $\left(\begin{array}{lll}0 & 1 & 0\end{array}\right)$ & 4425 & 1,54 \\
\hline 4 & $\left(\begin{array}{lll}0 & 1 & 1\end{array}\right)$ & 37074 & 12,25 \\
\hline 5 & $\left(\begin{array}{llll}1 & 0 & 0\end{array}\right)$ & 9092 & 3,08 \\
\hline 6 & $\left(\begin{array}{lll}1 & 0 & 1\end{array}\right)$ & 9750 & 3,19 \\
\hline 7 & $\left(\begin{array}{lll}1 & 1 & 0\end{array}\right)$ & 5382 & 1,76 \\
\hline 8 & $\left(\begin{array}{lll}1 & 1 & 1\end{array}\right)$ & 14199 & 1,81 \\
\hline
\end{tabular}

The fastest strategy (010) has processor time of 1.54 sec. Time gain is 1.8 times as compared with TSO.

Applying the methodology of the maximum principle based on formulas (8)-(16), it is possible to analyse the behaviour of function of Hamilton for all strategies of structural basis. The vectors of $\mathbf{X}$ and $\boldsymbol{\Psi}$ consist of six component in this case. The behaviour of function of Hamilton on an interval from 0 to $12 \mathrm{msec}$, for eight different values of the control vector, initial point $\mathbf{X}^{0}$ : $\left(x_{10}=0.0816, x_{20}=1.2039, x_{30}=0.7071, x_{40}=-2, x_{50}\right.$ $\left.=-1, x_{60}=-10\right)$ and the correct initial value of an auxiliary vector $\boldsymbol{\Psi}, \quad\left(\boldsymbol{\Psi}_{0 c}=(0.3,-1.85,-0.35,-1.9,0.32,2.5)\right)$ is presented in Fig. 9.

Value of $\boldsymbol{\Psi}_{0 c}$ has been obtained by the additional optimising procedure. Hamiltonian $H_{(111)}$ for strategy (111) has the greatest value from all eight strategies of the structural basis on the initial part of optimisation process from 0 to a point of $S_{\mathrm{w}}$ corresponding to the 36th step of integration of the system (4).

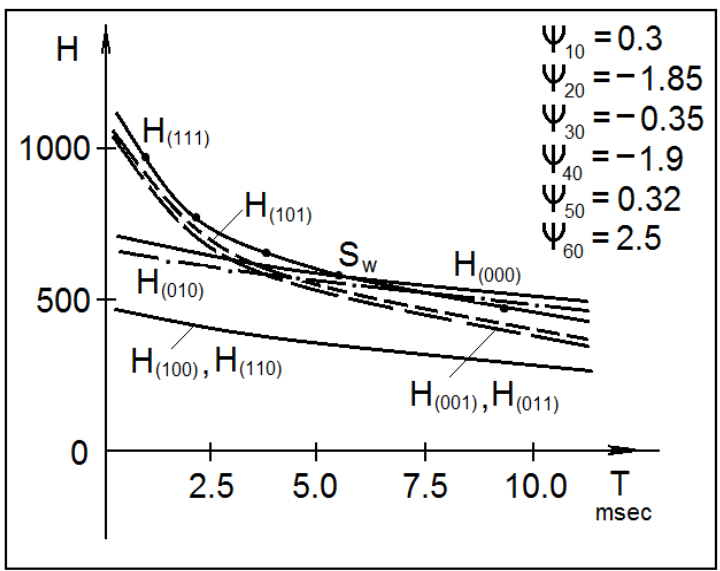

Fig. 9. Time dependency of functions $H_{(000)}, H_{(001)}, H_{(010)}$, $H_{(011)}, H_{(100)}, H_{(101)}, H_{(110)}, H_{(111)}$ for correct value of parameter $\boldsymbol{\Psi}_{0 c}=(0.3,-1.85,-0.35,-1.9,0.32,2.5)$

In this point, the Hamiltonian corresponding to TSO is equal to $H_{(111)}$ and then becomes greater. It means that since a point of $S_{\mathrm{w}}$, the optimum strategy is TSO. The point of switching of $S_{\mathrm{w}}$ appears automatically in the algorithm, and the behaviour of the function of Hamilton for all strategies of structural basis corresponds to Fig. 10.

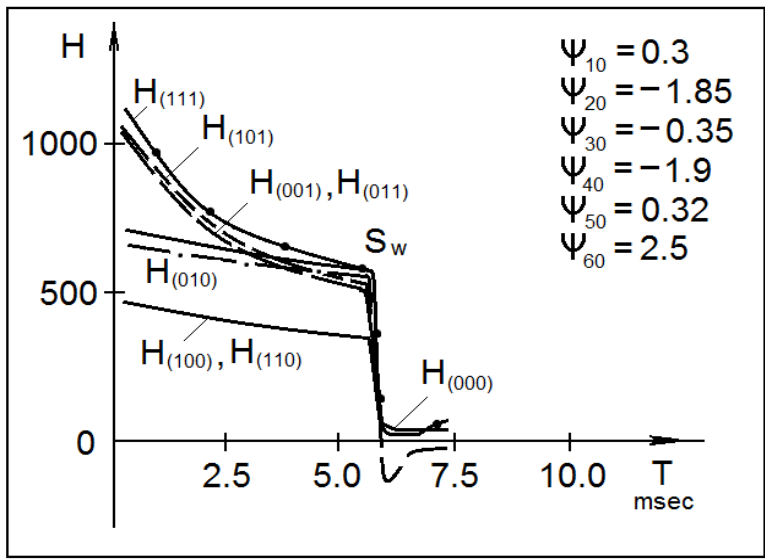

Fig. 10. Time dependency of functions $H_{(000)}, H_{(001)}, H_{(010)}$ $H_{(011)}, H_{(100)}, H_{(101)}, H_{(110)}, H_{(111)}$ for correct value of parameter $\boldsymbol{\Psi}_{0 c}$ and switch between $\boldsymbol{H}_{(111)}$ and $\boldsymbol{H}_{(000)}$

The $H_{(000)}$ function remains maximum until step 41, and then the $H_{(111)}$ function becomes maximum again and remains so until the end of the optimisation process. The time for optimisation is equal to $7.28 \mathrm{msec}$. The time gain of obtained optimum strategy in comparison with strategy (010) of Table 2 is equal to 211 times and 386 times in comparison with TSO.

The solution obtained above is not unique. It has turned out that by choosing other initial approximations for search of the correct initial value of an auxiliary vector $\boldsymbol{\Psi}$, it is possible to find other solutions also as in the previous example. 
Summarising the obtained results, it is possible to state two important facts. First, the theoretical result is obtained - theoretical justification is given for the earlier opened effect of acceleration of the process on optimisation of circuit in the conditions of a new methodology of design. This justification is based on the maximum principle.

Secondly, the analysis of the optimisation process of the presented circuits showed that application of the maximum principle really allows for the finding of the optimum structure of the control vector $\mathbf{U}(\mathrm{t})$ by means of an iterative procedure. Thus, considerable reduction of CPU time in comparison with traditional approach is observed when using the maximum principle. This result was obtained for the case of $\mathrm{N}$-dimensional space of parameters.

\section{CONCLUSIONS}

Analysis of the application of maximum principle to a problem of circuit optimisation proves that the formerly studied effect of acceleration on the process of optimisation appears owing to this principle. This means that the maximum principle of Pontryagin provides a theoretical justification for the acceleration effect that appears when we use the generalized formulation of process of circuit optimisation. It is confirmed that the maximum principle allows for finding one or several local minima of the functional that is defined as the processor time. Aside from that, the use of the maximum principle provides the chance to significantly reduce the time to circuit optimisation.

The analysis of optimisation process of the presented circuit showed that application of the maximum principle really allows finding the optimum structure of the control vector by means of iterative procedure. A new theoretical result is obtained. The possibility of applying this approach to optimizing large and complex circuits is the subject of further research.

\section{REFERENCES}

[1] Bunch J.R., Rose D.J. (Eds.). Sparse Matrix Computations. N.Y.: Academic Press, 1976.

[2] Osterby O., Zlatev Z. Direct Methods for Sparse Matrices. N.Y.: Springer-Verlag, 1983.

[3] Wu F.F. Solution of large-scale networks by tearing // IEEE Transactions on Circuits and Systems. 1976. V. 23. P. 706713.

[4] Sangiovanni-Vincentelli A., Chen L.K., Chua L.O. An efficient heuristic cluster algorithm for tearing large-scale networks // IEEE Transactions on Circuits and Systems. 1977. V. 24. P. 709-717.

[5] Rabat N., Ruehli A. E., Mahoney, G.W., Coleman, J.J. A survey of macromodeling // Proceedings of IEEE international symposium on circuits and systems. 1985. P. 139-143.

[6] Tadeusiewicz M., Kuczynski, A. (2013). A very fast method for the dc analysis of diode-transistor circuits // Circuits Systems and Signal Processing. 2013. V. 32. № 3, P. 433-451.
[7] Brayton R.K., Hachtel G.D., Sangiovanni-Vincentelli A.L. A survey of optimization techniques for integrated-circuit design // Proceedings of IEEE. 1981. V. 69. № 10. P. 13341362.

[8] Ruehli A.E. (Ed.). Circuit Analysis, Simulation and Design. V. 3. Amsterdam: Elsevier Science Publishers, 1987.

[9] Stehr G., Pronath M., Schenkel F., Graeb H., Antreich K. Initial sizing of analog integrated circuits by centering within topology-given implicit specifications // Proceedings of the IEEE/ACM international conference on computeraided design. 2003. P. 241-246.

[10] Hershenson M., Boyd S., Lee T. Optimal design of a CMOS op-amp via geometric programming // IEEE Transactions on Computer-Aided Design of Integrated Circuits and Systems. 2001. V. 20. № 1. P. 1-21.

[11] Kashirskiy I.S., Trokhimenko Y.K. General optimization for electronic circuits. Kiev: Tekhnika, 1979.

[12] Rizzoli V., Costanzo A., Cecchetti C. (1990, May 8-10). Numerical optimization of broadband nonlinear microwave circuits // Proceedings of IEEE MTT-S international symposium. 1990. V. 1. P. 335-338.

[13] Ochotta E.S., Rutenbar R.A., Carley L.R. (1996). Synthesis of high-performance analog circuits in ASTRX/OBLX // IEEE Transactions on Computer-Aided Design of Integrated Circuits and Systems. 1996. V. 15. P. 273-294.

[14] Zemliak A. Analog System Design Problem Formulation by Optimum Control Theory // IEICE Transactions on Fundamentals of Electronics Communications and Computer Sciences. 2001. V. E84-A. P. 2029-2041.

[15] Zemliak A.M. Comparative Analysis of the Lyapunov Function for Different Design Strategies of Analogue Circuits Design. // Radioelectronics and Communications Systems. 2008. V. 51. № 5. P. 233-238.

[16] Zemliak A., Markina T. Behavior of Lyapunov's function for different strategies of circuit optimization // International Journal of Electronics. 2015. V. 102. P. 619634.

[17] Pontryagin L.S., Boltyanskii V.G., Gamkrelidze R.V., Mishchenko E.F. The Mathematical Theory of Optimal Processes. N. Y.: Interscience Publishers, Inc., 1962.

[18] Neustadt L.W. Synthesis of time-optimal control systems. J. Math. Analysis and Applications. 1960. V. 1. № 2. P. 484 492.

[19] Rosen J.B. Iterative Solution of Nonlinear Optimal Control Problems // Journal of SIAM, Control Series A. 1966. P. 223-244.

[20] Fedorenko R.P. Priblizhennoe reshenie zadach optimalnogo upravleniya (Approximate Solution of Optimal Control Problems). Moscow: Nauka, 1978.

[21] Bourdin L., Trélat E. Pontryagin maximum principle for finite dimensional nonlinear optimal control problems on time scales // SIAM Journal on Control and Optimization. 2013. V. 51. № 5. P. 3781-3813.

[22] Zemliak A. Maximum principle for problem of circuit optimization // Electronics Letters. 2016. V. 52. № 9. P. 695-697.

[23] Zemliak A.M. (2002). Acceleration Effect of System Design Process // IEICE Transactions on Fundamentals of Electronics, Communications and Computer Sciences. 2002. V. E85-A, №7. P. 1751-1759.

[24] Massobrio G., Antognetti P. Semiconductor Device Modeling with SPICE. N.Y.: McGraw-Hill, 1993. 


\title{
О минимальном времени процесса оптимизации цепей
}

\author{
А.М. Земляк \\ Автономный университет Пуэбла, Мексика, Пуэбла \\ Национальный технический университет Украины, Украина, Киев azemliak@mail.ru
}

\begin{abstract}
Аннотация - Процесс оптимизации аналоговой схемы математически задан как управляемая динамическая система. В этом случае задачу минимизации времени проектирования можно сформулировать как задачу минимизации времени переходного процесса динамической системы. Специальный управляющий вектор, изменяющий структуру основных уравнений, служит инструментом для поиска лучших стратегий оптимизации. При этом принцип максимума Понтрягина является теоретическим подходом для нахождения оптимальной структуры управляющего вектора. Реализация принципа максимума основана на анализе поведения гамильтониана для различных стратегий оптимизации. Показано, что используя принцип максимума, возможно получить решение в форме локальных минимумов. Относительное ускорение процесса оптимизации цепи для лучшей стратегии, найденной на основе принципа максимума, составляет два - три порядка величины по сравнению с традиционным подходом. Получен новый теоретический результат. Применение данного подхода к оптимизации больших и сложных схем является предметом дальнейших исследований.
\end{abstract}

Ключевые слова - оптимизация цепей, управляемая динамическая система, стратегии оптимизации, принцип максимума Понтрягина.

\section{ЛИТЕРАТУРА}

[1] Bunch J.R., Rose D.J. (Eds.). Sparse Matrix Computations. N.Y.: Academic Press, 1976.

[2] Osterby O., Zlatev Z. Direct Methods for Sparse Matrices. N.Y.: Springer-Verlag, 1983.

[3] Wu F.F. Solution of large-scale networks by tearing // IEEE Transactions on Circuits and Systems. 1976. V. 23. P. 706713.

[4] Sangiovanni-Vincentelli A., Chen L.K., Chua L.O. An efficient heuristic cluster algorithm for tearing large-scale networks // IEEE Transactions on Circuits and Systems. 1977. V. 24. P. 709-717.

[5] Rabat N., Ruehli A. E., Mahoney, G.W., Coleman, J.J. A survey of macromodeling // Proceedings of IEEE international symposium on circuits and systems. 1985. P. 139-143.

[6] Tadeusiewicz M., Kuczynski, A. (2013). A very fast method for the dc analysis of diode-transistor circuits // Circuits Systems and Signal Processing. 2013. V. 32. № 3, P. 433-451.

[7] Brayton R.K., Hachtel G.D., Sangiovanni-Vincentelli A.L. A survey of optimization techniques for integrated-circuit design // Proc. of IEEE. 1981. V. 69. № 10. P. 1334-1362.

[8] Ruehli A.E. (Ed.). Circuit Analysis, Simulation and Design. V. 3. Amsterdam: Elsevier Science Publishers, 1987.
[9] Stehr G., Pronath M., Schenkel F., Graeb H., Antreich K Initial sizing of analog integrated circuits by centering within topology-given implicit specifications // Proceedings of the IEEE/ACM international conference on computeraided design. 2003. P. 241-246.

[10] Hershenson M., Boyd S., Lee T. Optimal design of a CMOS op-amp via geometric programming // IEEE Transactions on Computer-Aided Design of Integrated Circuits and Systems. 2001. V. 20. № 1. P. 1-21.

[11] Kashirskiy I.S., Trokhimenko Y.K. General optimization for electronic circuits. Kiev: Tekhnika, 1979.

[12] Rizzoli V., Costanzo A., Cecchetti C. (1990, May 8-10). Numerical optimization of broadband nonlinear microwave circuits // Proceedings of IEEE MTT-S international symposium. 1990. V. 1. P. 335-338.

[13] Ochotta E.S., Rutenbar R.A., Carley L.R. (1996). Synthesis of high-performance analog circuits in ASTRX/OBLX // IEEE Transactions on Computer-Aided Design of Integrated Circuits a nd Systems. 1996. V. 15. P. 273-294.

[14] Zemliak A. Analog System Design Problem Formulation by Optimum Control Theory // IEICE Transactions on Fundamentals of Electronics Communications and Computer Sciences. 2001. V. E84-A. P. 2029-2041.

[15] Zemliak A.M. Comparative Analysis of the Lyapunov Function for Different Design Strategies of Analogue Circuits Design. // Radioelectronics and Communications Systems. 2008. V. 51. № 5. P. 233-238.

[16] Zemliak A., Markina T. Behavior of Lyapunov's function for different strategies of circuit optimization //International Journal of Electronics. 2015. V. 102. P. 619-634.

[17] Pontryagin L.S., Boltyanskii V.G., Gamkrelidze R.V., Mishchenko E.F. The Mathematical Theory of Optimal Processes. N. Y.: Interscience Publishers, Inc., 1962.

[18] Neustadt L.W. Synthesis of time-optimal control systems. J. Math. Analysis and Appl. 1960. V. 1. № 2. P. 484-492.

[19] Rosen J.B. Iterative Solution of Nonlinear Optimal Control Problems // Journal of SIAM, Control Series A. 1966. P. 223-244.

[20] Федоренко Р.П. Приближенное решение задач оптимального управления. М.: Наука, 1978.

[21] Bourdin L., Trélat E. Pontryagin maximum principle for finite dimensional nonlinear optimal control problems on time scales // SIAM Journal on Control and Optimization. 2013. V. 51. № 5. P. 3781-3813.

[22] Zemliak A. Maximum principle for problem of circuit optimization // Electronics Letters. 2016. V. 52. № 9. P. 695-697.

[23] Zemliak A.M. (2002). Acceleration Effect of System Design Process // IEICE Transactions on Fundamentals of Electronics, Communications and Computer Sciences. 2002. V. E85-A, №7. P. 1751-1759.

[24] Massobrio G., Antognetti P. Semiconductor Device Modeling with SPICE. N.Y.: McGraw-Hill, 1993. 